\section{Measuring SPM Piezo \\ Displacement Responses}

Heinz Sturm*, Markus Heyde ${ }^{* *}$ and Klaus Radernann ${ }^{* \star}$

*Federal Institute of Materials Research (BAM), Berlin

** Institute of Physical Chemistry, Humboldt University, Berlin

\section{Introduction}

Scanning Probe Microscopy (SPM) is a versatile tool for the investigations of surfaces. Additionally to the three-dimensional examination of surface topography, local mechanical or electrical properties can be measured ${ }^{1}$. In this work we present a short contribution on the performance and reliability of piezoelectric transducers in a SPM, which take care of the fine positioning of the tip relative to the sample. Some of the calibration procedures known in literature [cited in 2] are: laser interferometry, scanning a slightly tilted surface, a grid, a crystallographic or artificial step of a known height or by using a reference piezo. To measure slow and fast piezo response simultaneously, we use a non-contact calibration procedure with a high dynamic range (Ångstrom to several hundred micrometers) and high frequency range (D.C. to $200 \mathrm{kHz}(-3 \mathrm{~dB})$ ). Hysteresis of the piezo displacement can reach up to $20 \%$ of total elongation. So, for the $z$ piezo which has to follow ascending and descending topographical steps and the sample tilt, hysteresis should be regarded as the most important source of errors. Furthermore, the displacement depends on the voltage pre-history, so often SPM data are collected in one lateral direction to minimize the divergence between the lateral commanded position and the true position. After all, the piezo response may suffer from creep and aging with time or temperature.

The aim of this work is to measure the static and dynamic movement of a $z$ piezo. The collected data can be used to characterize separately the non-linearity and hysteresis for slow and fast z-displacements as well. Additionally, the problematic situation of a sample tilt (requiring slow but large zdisplacement) superimposed by small topographic features (requiring fast but small z-displacement) is analyzed.

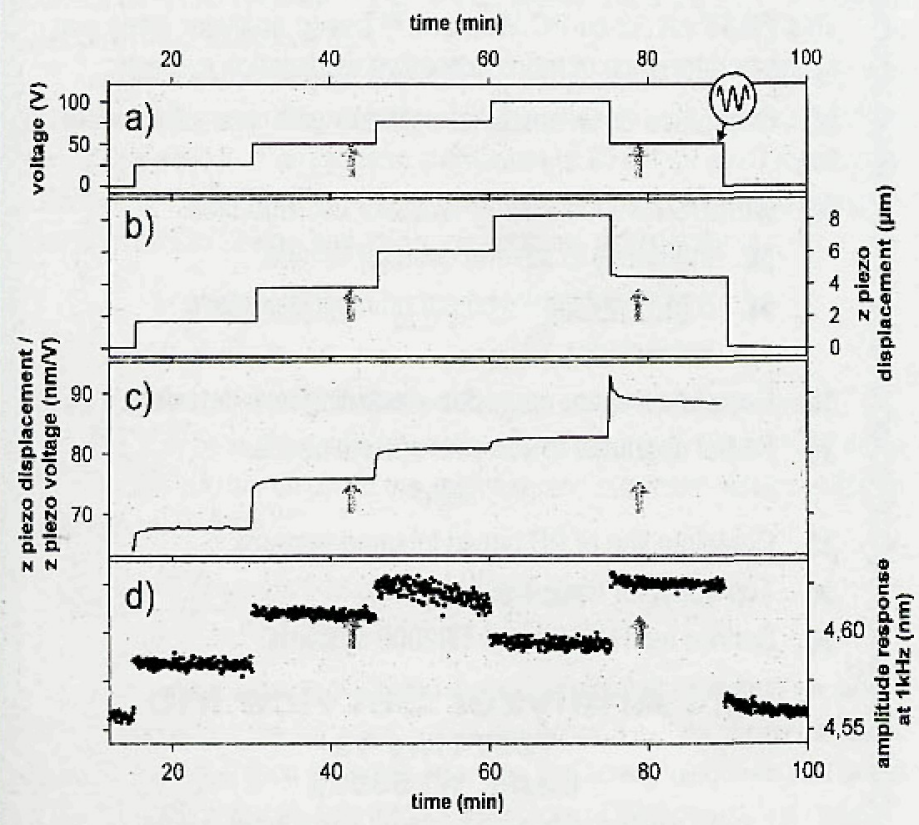

Figure 1: a) Stepped piezo voltage excitation superimposed with $1 \mathrm{~V}$ at $1 \mathrm{kHz}$ (TRI piezo), b) Absolute piezo displacement, c) Relative piezo displacement, d) dynamic response at $1 \mathrm{kHz}$.

\section{Experiments and Results}

In the further experiments discussed in this article, the $z$-direction of a tripod scanner (TRI) and tube scanner (TUB) have been examined. The TRI ${ }^{3}$, is made from three discrete piezos for $x, y$ and $z$, mounted orthogonal. The TUB is made from a hollow cylinder of piezoelectric ceramic which is commercially. available ${ }^{4}$. The outside of the TUB is supplied with four separate electrodes, the inner surface of the cylinder forms a continuous counter-electrode. By applying a voltage to the outer electrodes, the z-movement of the scanner is performed.

The experimental set-up is described in detail elsewhere? 2 . The displacement sensor ${ }^{5}$ utilizes two bundles of glass fibers to illuminate a surface and to receive and measure the reflected light intensity from a surface. At the sensor tip, non-collimated light rays diverge outward from each fiber in a conical light path. The intensity of the reflected light leads to an output voltage with a sensitivity of $109.14 \mathrm{mV} / \mu \mathrm{m}$. To determine the dynamic response of the piezos, an A. C. signal of $1 \mathrm{~V}$ at $1 \mathrm{kHz}$ is superimposed to the D.C. voltage. These conditions are chosen to represent small topographic features, i.e., 1000 particles on a $1000 \mathrm{~nm}$ scan line with $1000 \mathrm{~nm} / \mathrm{s}$ scan velocity. The dynamic displacement of the piezos is about $4.5 \mathrm{~nm} / \mathrm{V}$ for the TRI and $0.65 \mathrm{~nm} / \mathrm{V}$ for the TUB, respectively. The A.C. voltages are measured with a lock-in amplifier.

At first, the step response of the TRI has been evaluated measuring the absolute (Figure 1b) and the relative (Figure 1c) D.C. displacement response and the simultaneously performed dynamic response (Figure 1d). At $50 \mathrm{~V}$ (marked with arrows) a strong hysteresis can be observed. Dividing the piezo displacement by the used voltage, more effects are visible. At $50 \mathrm{~V}$ the history dependent deviation is about $12.7 \mathrm{~nm} / \mathrm{V}$ (i.e. $635 \mathrm{~nm}$ ) even after $15 \mathrm{~mm}$. Creep can be observed after a voltage change. If the voltage sign remains unchanged, the creep can be regarded as zero after 15 minutes settling time. Accepting an error of some percent, the required settling time may be regarded as 2 to 4 minutes. With increasing woltage, saturation takes place. After a voltage step back to $50 \mathrm{~V}$, a strong deviation is observed, caused by hysteresis as a dead band phenomenon. The amplitude response at $1 \mathrm{kHz}$ (Figure 1d) shows several important effects: $i$. The influence of the D.C. voltage is clearly visible and will lead to topography errors in a SEM image if an instantaneous change of the voltage takes place at topography steps. At $100 \mathrm{~V}$ a saturation effect can be noticed. ii. A time dependent arm is found, which might hinder the interpretation of kinetic investigations. iif. At $75 \mathrm{~V}$, an increased drift as well as an unexpected noise can be observed.

The sample tilt analysis is carried out using $0.3 \mathrm{~V} / \mathrm{s}$ (Figures $2-4$ ). The virgin curves are not given, up to 10 cycles are necessary for drift-free, i.e. closed

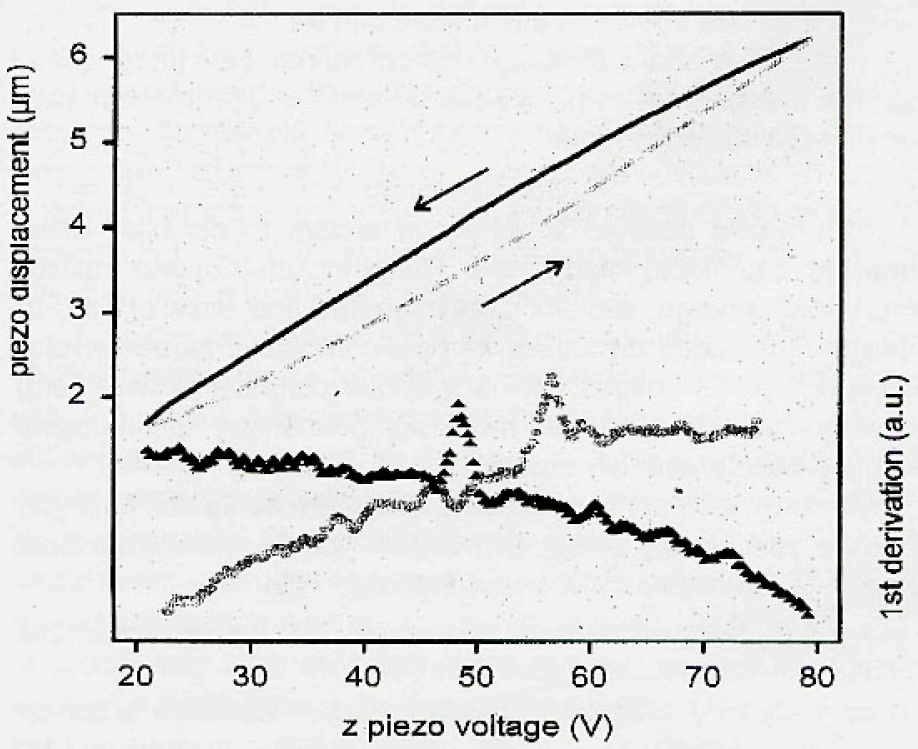

Figure 2: Piezo response on a triangular wave voltage cycle (25 nm/s steady state) and the first derivation of both branches, TRI piezo. 
hysteresis loops. The data of the piezo displacement can be split (Figures 2 and 3 ) into two branches for increasing and decreasing D.C. voltage. The first derivation (figure 2, TRI piezo) is calculated after a slight suppression of the high frequency noise. Irregularities are found at $50 \mathrm{~V}$ and $60 \mathrm{~V}$ depending on the voltage pre-history. The reason for this reproducible result is not attributed to a saturation effect, because the selected voltage span corresponds to only $60 \%$ of the ffull scale elongation.

The TUB piezo does not show these spontaneous displacement jumps. The average of both branches (Figure 3a) give a hysteresis-free but not exactly linear curve (Figure 3b). Saturation effects are visible (insets). This non-linearity data is fitted with a fifth order polynomial without the even coefficients. Subtracting the non-linearity from the two original displacement branches gave the pure hysteresis (Figure 3c), which are both fitted to a sixth order polynomial without the odd coefficients. Obviously, the even coefficients describing the hysterosis change the sign with the direction of the piezos' voltage change. Using the values for $d_{1}$ to $d_{6}$ together with the probe gap depended offsets, two branches of the piezo motion can be calculated, the residuals (Figure $3 \mathrm{~d}$ ) describe the misfit between measured and calculated data and follow a second order polynomial. Though the misfit was calculated by subtracting the synthesized from the measured data, the curvature at high voltages means that independently from the sign of the voltage the data values of the synthetic curve are too small. Though this

a)
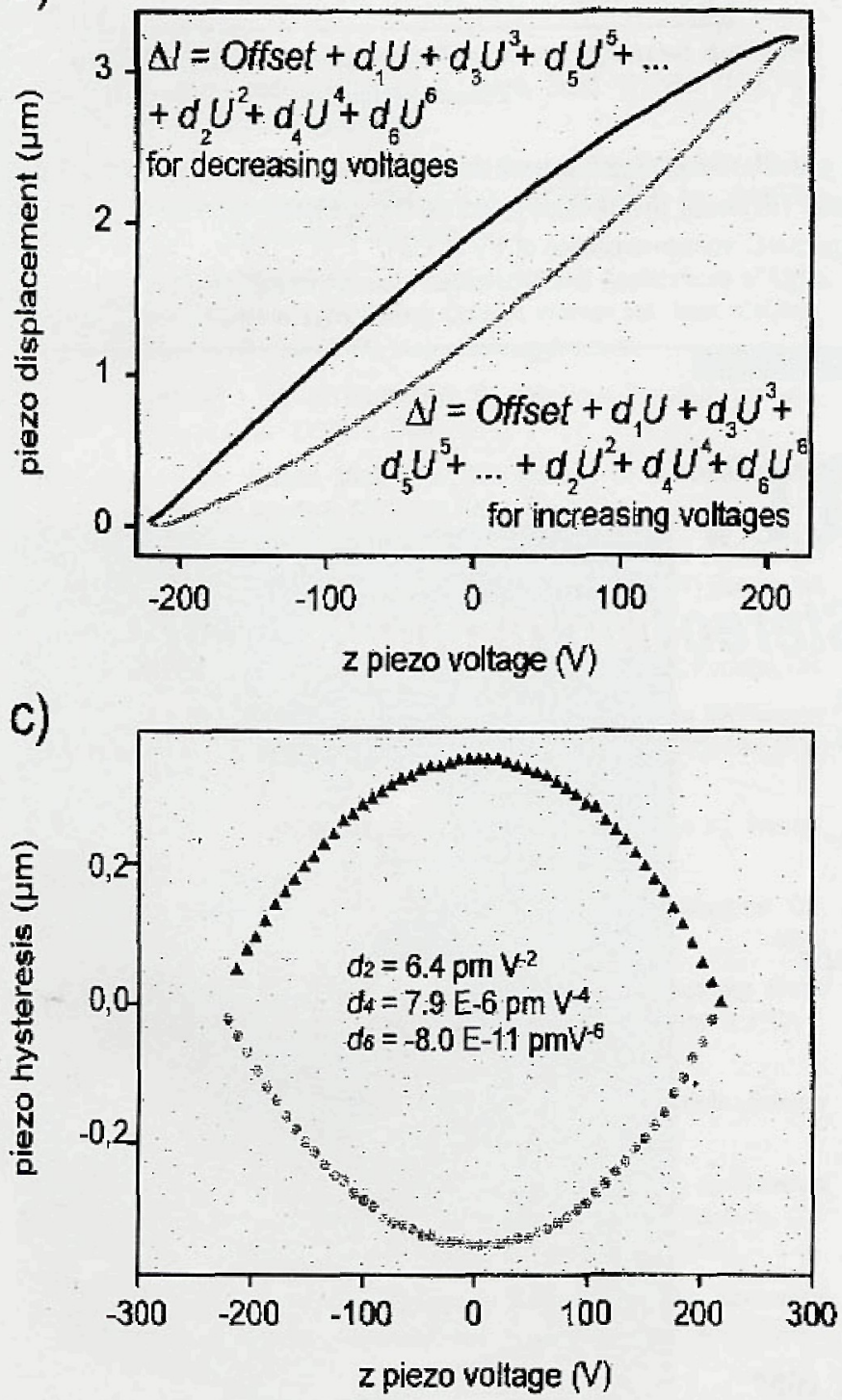

additional extension of the piezo is a quadratic function of the voltage, it should be electrostriction. The importance of the high order coefficients gets more concise if they are multiplied with a specific voltage $(e . g ., 100 \mathrm{~V}): \mathrm{d}_{4} \cdot U 4:=790 \mathrm{pm}$, $d_{5} \cdot U_{5}=580 \mathrm{pm}$ and $\mathrm{d}_{6} \cdot U^{6},=80 \mathrm{pm}$.

The last experiment presented corresponds to a SPM experiment with a tilted surface plane (Figure 4, TRI piezo) and topographic features ( $1 \mathrm{~V}$ at 1 $\mathrm{kHz}$ ). The measured dynamic piezo response (Figure $4 \mathrm{~b}$ ) shows a remarkable hysteresis and non-linearity. The topography error in a SPM image would reach $15 \%$, assuming particles or steps of about $4.4 \mathrm{~nm}$ height. The phase shift (Figure 4c) between the A.C. voltage and the piezo displacement is not constant. During scanning, this could lead to a local change of the constant force (SFM) or constant current (STM) feedback or to changes of the measured local stiffness in a force modulation experiment.

\section{Conclusion}

Measurements of the displacement response of two SPM piezos using a fiber optical displacement sensor show the usefulness of controlling their performance. The uncertainty of SPM images containing topography steps or a tilted surface can be assessed. It is demonstrated that the analysis of small topographic features at interfaces or clusters in the range of nanometer or below

\section{Continued on following page}

b)

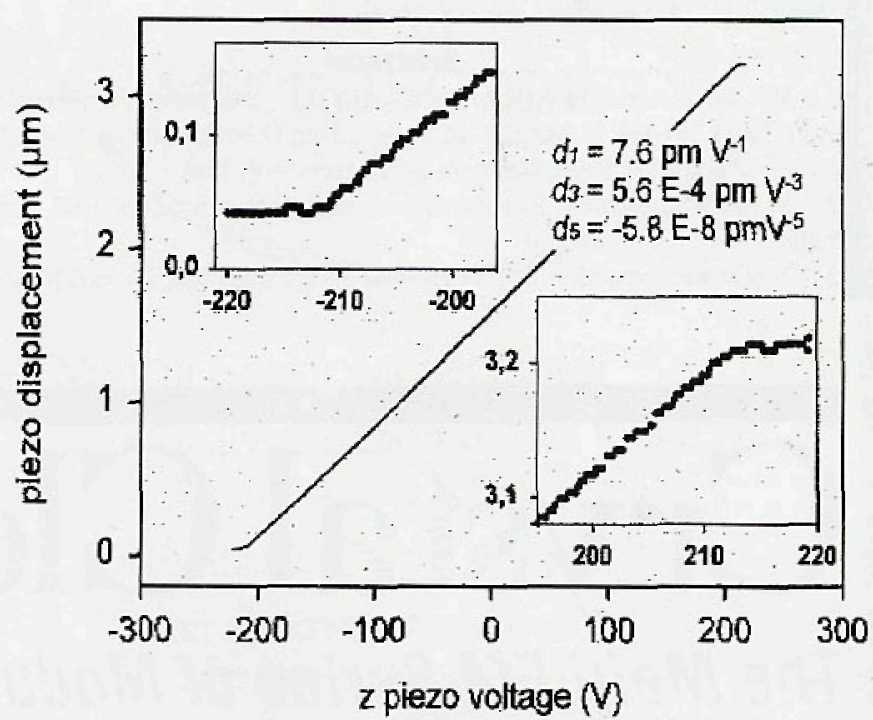

d)

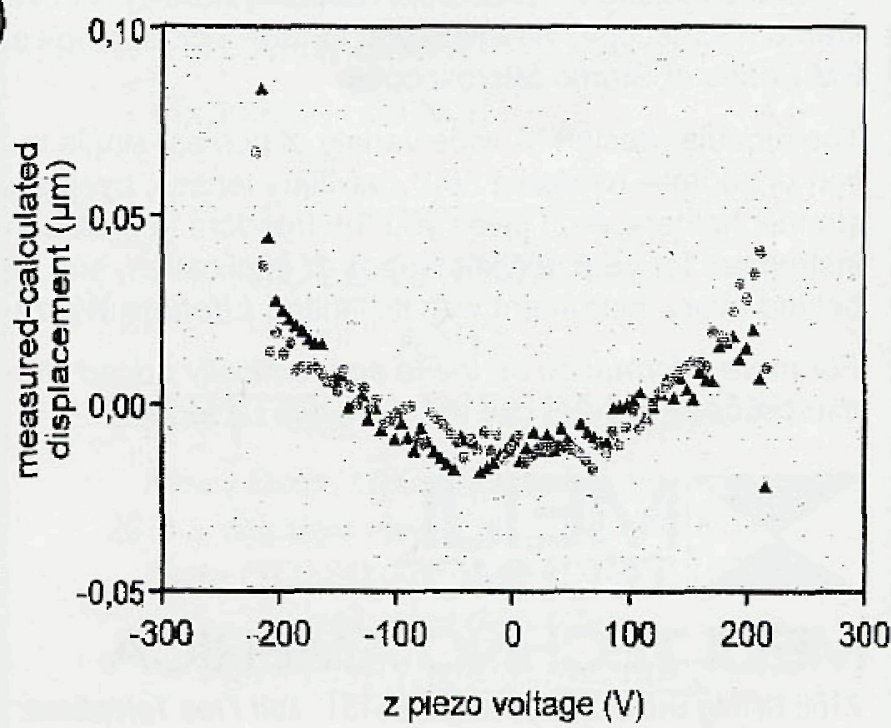

Figure 3: a) Piezo response on a triangular wave voltage cycle (2 nm/s steady state, TUB piezo), b) Piezo non-linearity, c) Piezo hysteresis, d) Residuals showing the electrostrictive effect. For $\mathrm{a}, \mathrm{c}$ and $\mathrm{d}$ : Grey: increasing voltage, black: decreasing voltage. 


\section{Measuring SPM Piezo Displacement Responses}

Continued from preceding page

suffer from the voltage dependent history of the piezo displacement. Especially the measured height of a particle or step on a flat but tilted surface can be faked by remarkable errors. A change of the $z$ position of a sample due to $a$ readustment of the tilt or due to a simple change or the location of interest are connected with a change in the static elongation of the $z$ piezo. So, the consequence for high resolution SPM measurements is that even a small change of the $z$ position can give unexpected results and can lead to misinterpretation. It should be mentioned that the two examined piezos can be regarded as typical for the two different transducer techniques and not for the piezo quality of a specific manufacturer. It has to be expected that piezoelectric transducers (open-loop systems) of all commercial or home-built SPMs show the same tendency. The often discussed subjective impression that the performance of the SPM feedback depends on the z piezo offset is confirmed.

\section{Acknowledgements}

Many thanks to M. Munz for fruitful discussions and to R. Sernow for the technical support. This work was part of the research co-operation program involving the Humboldt University and the Federal Institute of Materials Research and Testing. The financial support of the German Science Foundation within the Sfb 605 "Elementare Reibereignisse" (H. S.) and project DFG RaA94/8-1 (M.H.) is gratefully acknowledged.

\section{References}

1. V.P. Bovtoun, H. Sturm, M.A. Leshichenko, Yu. I. Yakimenko, Ferroelectrics 190 (1-4) (1997), 161-166; M. Munz, H. Sturm, E. Schulz, O. Hinrichsen, Composites A 29A (1998), 125l-1259; H. Strum, Macromol Symp, subm. Aug. 1998

2. M. Heyde, H. Sturm, K. Rademann, Surf. Interf. Anal, subin. Sept. 1998, acc. Oct. 1998

3. TopoMetrix Corporation, 5403 Betsy Ross Drive, Santa Clara, CA 95054-1192.
4. PI Ceramic GmbH, LindenstraBe, D-07589 Lederhose, ERG.

5. Model D20-A1-H Fibre Optic Displacement Sensor, Philtec, Inc., P.O. Box 359 Arnold, MD 21012, U.S.A.

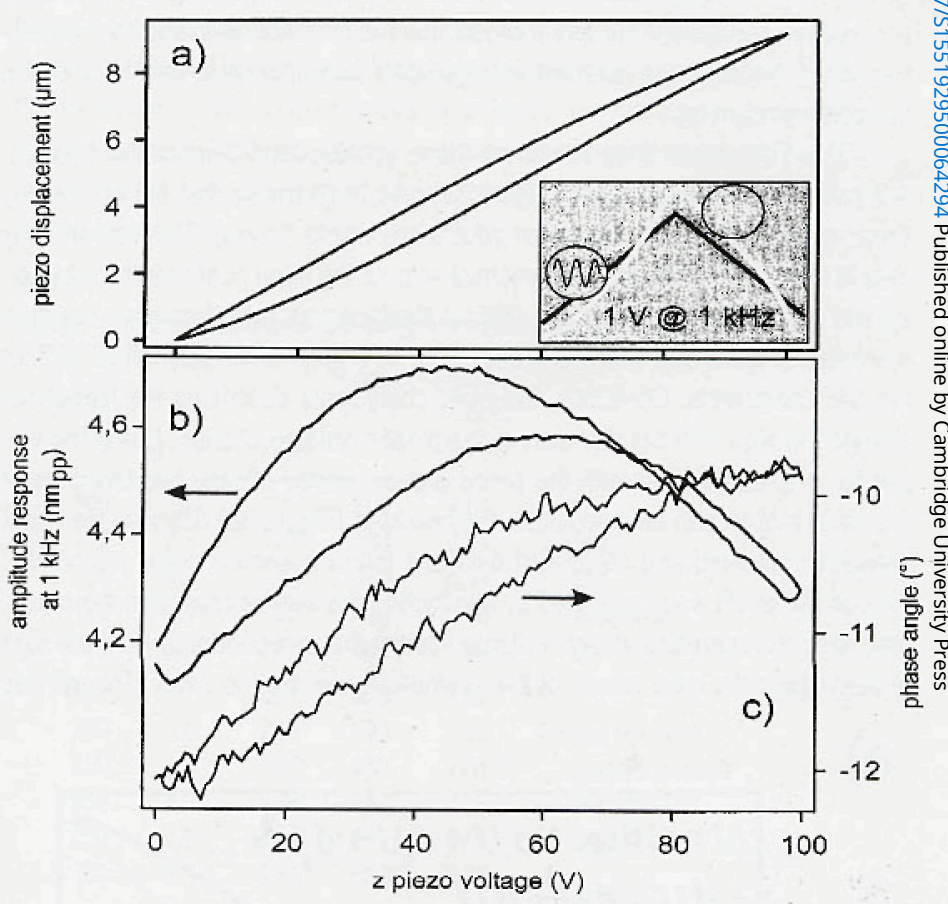

Figure 4: a) Piezo response on a triangular wave voltage cycle ( $25 \mathrm{~nm} / \mathrm{S}$ steady state, TRI piezo), b) Amplitude response and c) Phase response on the superimposed A.C. voltage excitation of $1 \mathrm{~V}$ at $1 \mathrm{kHz}$.

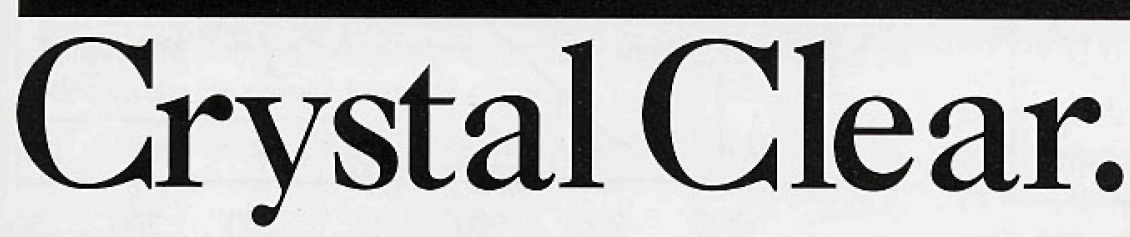

\section{The Meiji EM Series of Modular Stereo Mieroscopes.}

If you are looking for precision, durability, quality and value in a stereo microscope, we invite you to take a closer look at Meiji's EM Series of Stereo Microscopes.

The modular design (A wide variety of bodies, single magnification or zoom- rotatable $360^{\circ}$, auxiliary lenses, eyepieces, stands, holders, etc.) gives you the freedom to create the ideal instrument for your specific needs or application, and Meiji stands behind every instrument with its limited Lifetime Warranty.

For more information on these economically priced stereo microscopes, please call, FAX or write us today.

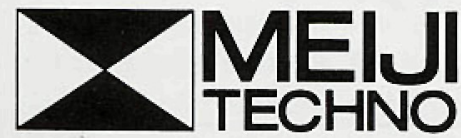

\section{MEIJI TECHNO AMERICA}

2186 Bering Drive, San Jose, CA 95131, Toll Free Telephone: $\mathbf{8 0 0 . 8 3 2 . 0 0 6 0}$ FAX: 408.428.0472, Tel: 408.428 .9654 